\title{
Rendimiento productivo de pollos parrilleros alimentados con harina de yuca (Manihot esculenta) como reemplazo del maíz
}

\author{
Productive performance of broilers fed cassava meal (Manihot esculenta) \\ as maize replacement
}

\author{
William Celis P. ${ }^{1,2}$, Marco Mathios F. ${ }^{1}$, Jorge Cáceres C. ${ }^{1}$, José Aguilar V. ${ }^{1}$
}

\section{Resumen}

El ensayo tuvo por objetivo determinar el rendimiento productivo de pollos parrilleros alimentados con harina de yuca (Manihot esculenta) en reemplazo parcial del maíz en la etapa de acabado. Se utilizaron 96 pollos parrilleros de la línea Cobb de 21 días de edad, distribuidos en 12 corrales experimentales correspondientes a tres tratamientos con cuatro repeticiones. Los niveles de sustitución del maíz por harina de yuca fueron de $0,10 \mathrm{y}$ $20 \%$, los que representaron a los tratamientos T0 (testigo), T1 y T2, respectivamente. Cada tratamiento tuvo 32 pollos y cada repetición ocho pollos. Se evaluó el efecto de la harina de yuca en el consumo de alimento, incremento de peso, conversión alimenticia, mortalidad e índice de eficiencia productivo. No se encontraron diferencias significativas entre tratamientos en los indicadores evaluados, lo que indica que la harina de yuca puede reemplazar eficazmente hasta un $20 \%$ en las dietas basadas en el maíz para pollos de engorde.

Palabras clave: harina de yuca; maíz; pollos parrilleros

\section{Abstract}

The aim of this study was to determine the productive performance of broilers fed cassava meal (Manihot esculenta) as partial replacement of maize in the finishing stage. A total of 96 broiler chickens of the 21-day-old Cobb line were used, distributed in 12 experimental pens corresponding to three treatments with four repetitions. The substitution levels of maize for cassava meal were 0,10 and $20 \%$, which represented the treatments T0

Facultad de Zootecnia, Universidad Nacional Autónoma de Alto Amazonas, Amazonas, Perú E-mail: wcelis@unaaa.edu.pe

Recibido: 13 de marzo de 2018

Aceptado para publicación: 15 de enero de 2019 
(control), T1 and T2, respectively. Each treatment had 32 chickens and each repetition eight chickens. The effect of cassava flour on feed consumption, weight gain, feed conversion, mortality and productive efficiency index was evaluated. No significant differences were found between treatments in the evaluated indicators, indicating that cassava meal can effectively replace up to $20 \%$ in broiler maize-based diets.

Key words: cassava flour; corn; broiler chickens

\section{INTRODUCCIÓN}

El costo de producción del kilogramo de pollo parrillero en la ciudad de Yurimaguas y, en general, en el departamento de Loreto, Perú, es alto, comparado con las demás regiones del país, debido principalmente a los elevados precios de los insumos que se usan en la alimentación de los pollos. El maíz constituye el ingrediente principal de las raciones para aves, con más del $60 \%$ dentro de las fórmulas alimenticias, y cuya producción en la zona es reducida y estacional, alcanzándose rendimientos de 1.94 toneladas por hectárea (Cubas et al., 2009), dado a que se practica una agricultura tradicional bajo condiciones de suelos ácidos y poco fértiles. Esta condición origina una demanda insatisfecha permanente de este grano y, por lo tanto, un precio elevado en el mercado local. El costo del alimento representa el $65-75 \%$ del presupuesto para la cría de las aves (Gonzales, 2014).

Por lo descrito, se requiere evaluar insumos alternativos al maíz, como podría ser la yuca (Manihot esculenta), por ser un cultivo adaptado a las condiciones de clima y suelo de la zona y su cosecha es continua durante todo el año, con producciones de 13.2 toneladas por hectárea (Almeida y Ferreira, 2005). Esta característica permite tener menos costo que el maíz y con valores energéticos considerables $-3138 \mathrm{kcal} / \mathrm{kg}$ de energía metabolizable (Rostagno et al., 2011), 88.5\% de materia seca, 3.0 de proteína bruta y $6.0 \%$ de fibra (Silva et al., 2015). Por tal razón, la yuca constituye un alimento con gran poten- cial para ser utilizado como alternativa en la alimentación animal, debido principalmente a su valor energético (Cagnon et al., 2002).

Sin embargo, la yuca presenta factores antinutricionales, como los polisacáridos no amiláceos, que provocan menor eficiencia, menor ganancia de peso y disminución de la digestibilidad de diversos nutrientes (Brufau et al., 1994), además de la toxicidad del ácido cianhídrico, aunque estos elementos son desactivados cuando la yuca es sometida a calor. El objetivo del presente trabajo fue determinar el efecto sobre los índices zootécnicos de los pollos parrilleros cuando son alimentados con harina de yuca en reemplazo del maíz.

\section{MATERIALES Y Métodos}

El estudio se llevó a cabo en el campus de la Universidad Nacional Autónoma de Alto Amazonas, situado en la ciudad y distrito de Yurimaguas de la región Loreto, Perú, en el año 2016. Se utilizó un galpón de 16 x 5.5 m, construido con material noble. En su interior se construyeron 12 corrales experimentales de $1 \mathrm{~m}^{2}$ con listones de madera aserrada de 2 x 2" de espesor. Se contó con 96 pollos parrilleros machos de la línea Cobb 500 de 21 días de edad.

En los primeros 21 días los pollos recibieron una dieta comercial de inicio con $22 \%$ de proteína y $3000 \mathrm{kcal} / \mathrm{kg}$ de energía metabolizable. A los 21 días, día de inicio del 
experimento, y hasta los 40 días consumieron tres dietas con diferentes niveles de harina de yuca en reemplazo del maíz, las mismas que fueron suministradas ad libitum y ajustadas a los requerimientos nutricionales recomendadas por Rostagno et al (2011) para pollos parrilleros ( $18 \%$ de proteína y 3200 $\mathrm{kcal} / \mathrm{kg}$ de energía metabolizable, aproximadamente, en todas las raciones). En el Cuadro 1 se muestra la fórmula alimenticia y el valor nutricional de las raciones mencionadas.

Se utilizaron tres tratamientos con cuatro repeticiones, donde los tratamientos $\mathrm{T} 0$ (testigo), T1 y T2 tenían 0,10 y $20 \%$ de harina de yuca. En el Cuadro 2 se indican los valores del análisis proximal de las dietas experimentales. Cada tratamiento tuvo 32 pollos y cada repetición ocho pollos. Un corral experimental representó a cada una de las repeticiones (unidad experimental). Se registró el consumo diario por tratamiento mediante la diferencia entre lo suministrado y el residuo del alimento en 24 horas, mientras que el consumo acumulado se obtuvo con la suma de los consumos de las tres semanas que duró la fase experimental. El alimento fue pesado en una balanza electrónica de $30 \mathrm{~kg}$ de capacidad y $5 \mathrm{~g}$ de precisión.

Los pollos fueron pesados semanalmente en la misma balanza del alimento. La diferencia del peso corporal de la semana actual menos el peso de la semana anterior constituyó el incremento de peso semanal. El incremento acumulado se obtuvo sumando los incrementos de las tres semanas. La conversión alimenticia por tratamiento se obtuvo mediante el alimento consumido y el peso del pollo. Además, se determinó el índice de eficiencia productivo europeo, mediante el producto del peso vivo $(\mathrm{kg})$ con la viabilidad (\%) x 100 , dividido entre el producto de la edad de saca (días) con la conversión alimenticia $(\mathrm{kg} / \mathrm{kg})$. La mortalidad se registró diariamente en cada tratamiento y fue expresada en forma porcentual.
Para el análisis estadístico se utilizó un diseño completamente al azar y para la comparación entre las medias de los tratamientos se aplicó la prueba de Duncan $(\mathrm{p}<0.05)$. Se utilizó el programa estadístico Lenguaje R.

\section{Resultados y Discusión}

Los resultados del ensayo se encuentran en el Cuadro 3. El incremento de peso ( $\mathrm{kg} / \mathrm{ave}$ ) fue uniforme, no habiendo diferencias estadísticas. Los valores obtenidos evidencian que la harina de yuca no tuvo efecto negativo sobre el incremento de peso de las aves. Estos resultados concuerdan con el trabajo de Ferreira (2010), quien reportó que niveles de hasta $20 \%$ de harina de yuca no afectan el rendimiento productivo de los pollos. Contrariamente Valdivié et al. (2008) y Nascimento et al. (2005) reportaron rendimientos menores conforme se incrementaba el nivel de yuca en la ración, posiblemente debido a la variedad de yuca utilizada. Se conoce que el nivel de fibra y las concentraciones de factores anti-nutricionales varían entre las variedades de yuca (Brufau et al., 1994).

No hubo diferencias significativas en el consumo de alimento (kg/ave), tal y como reporta Valdivié et al. (2008), aunque Nascimento et al. (2005) indican que el consumo decrece conforme aumentan los niveles de yuca en la ración. Es importante hacer notar que harina de yuca muy fina produce apelmazamiento del alimento en el pico del ave, lo que dificulta el consumo. La partícula gruesa en la alimentación está asociada a un buen desarrollo intestinal, mejorando la función de la molleja y reduciendo problemas proventriculares (Jones y Taylor, 2001). Con base a este fundamento, en el presente estudio se utilizó un tamaño de partícula de 0.7 mm aproximadamente. 
Cuadro 1. Fórmula alimenticia y valor nutricional de las dietas del estudio

\begin{tabular}{lccc}
\hline \multirow{2}{*}{ Ingrediente } & \multicolumn{3}{c}{ Nivel de sustitución (\%) } \\
\cline { 2 - 4 } & 0 & 10 & 20 \\
\hline Maíz chancado & 69.50 & 62.55 & 55.60 \\
Torta de soya & 21.66 & 20.96 & 17.96 \\
Harina de pescado & 4.00 & 4.50 & 7.50 \\
Aceite de palma & 2.00 & 2.20 & 2.20 \\
Harina de yuca & 0.00 & 6.95 & 13.90 \\
Carbonato de calcio & 1.10 & 1.10 & 1.10 \\
Fosfato monocálcico & 0.80 & 0.80 & 0.80 \\
Metionina & 0.30 & 0.30 & 0.30 \\
Cloruro de colina & 0.20 & 0.20 & 0.20 \\
Sal común & 0.20 & 0.20 & 0.20 \\
Premix & 0.10 & 0.10 & 0.10 \\
Fungicida & 0.05 & 0.05 & 0.05 \\
Bac zinc & 0.05 & 0.05 & 0.05 \\
Coccidiostato & 0.04 & 0.04 & 0.04 \\
\hline Total & 100 & 100 & 100 \\
\hline Nutrientes calculados & & & \\
Energía metabolizable (Mcal/kg) & 3.2 & 3.2 & 3.2 \\
Nutrientes analizados ${ }^{1}$ & & & \\
Proteína (\%) & 17.46 & 18.16 & 18.34 \\
\hline
\end{tabular}

${ }^{1}$ Laboratorio de Evaluación Nutricional de Alimentos, Universidad Nacional Agraria La Molina, Perú

Cuadro 2. Análisis proximal de las dietas experimentales ${ }^{1}$

\begin{tabular}{ccccccc}
\hline Tratamiento $^{2}$ & $\begin{array}{c}\text { Humedad } \\
\%\end{array}$ & $\begin{array}{c}\text { Grasa } \\
\%\end{array}$ & $\begin{array}{c}\text { Fibra cruda } \\
\%\end{array}$ & $\begin{array}{c}\text { Ceniza } \\
\%\end{array}$ & $\begin{array}{c}\text { Proteína } \\
\%\end{array}$ & $\begin{array}{c}\text { ELN }^{3} \\
\%\end{array}$ \\
\hline T0 & 11.68 & 4.62 & 2.08 & 3.64 & 17.46 & 60.52 \\
T1 & 11.19 & 5.69 & 2.02 & 4.53 & 18.16 & 58.41 \\
T2 & 11.12 & 5.82 & 1.91 & 4.90 & 18.34 & 57.91 \\
\hline
\end{tabular}

${ }^{1}$ Laboratorio de Evaluación Nutricional de Alimentos, Universidad Nacional Agraria La Molina, Lima

${ }^{2}$ TO, T1, T2: 0, 10 y $20 \%$ de harina de yuca en la dieta

${ }^{3}$ Extracto libre de nitrógeno 
Cuadro 3. Parámetros productivos de pollos parrilleros alimentados con diferentes niveles de reemplazo de maíz con harina de yuca

\begin{tabular}{lccc}
\hline Parámetro & T0 & T1 & T2 \\
\hline Peso vivo inicial, kg/ave & $0.80^{\mathrm{a}} \pm 0.02$ & $0.81^{\mathrm{a}} \pm 0.02$ & $0.80^{\mathrm{a}} \pm 0.02$ \\
Peso vivo final, kg/ave & $2.17^{\mathrm{a}} \pm 0.10$ & $2.17^{\mathrm{a}} \pm 0.08$ & $2.19^{\mathrm{a}} \pm 0.06$ \\
Incremento de peso, $\mathrm{kg} / \mathrm{ave}$ & $1.37^{\mathrm{a}} \pm 0.10$ & $1.36^{\mathrm{a}} \pm 0.06$ & $1.39^{\mathrm{a}} \pm 0.07$ \\
Consumo de alimento, $\mathrm{kg} / \mathrm{ave}$ & $2.78^{\mathrm{a}} \pm 0.26$ & $2.71^{\mathrm{a}} \pm 0.09$ & $2.82^{\mathrm{a}} \pm 0.05$ \\
Conversión alimenticia kg/kg & $2.02^{\mathrm{a}} \pm 0.09$ & $1.99^{\mathrm{a}} \pm 0.05$ & $2.05^{\mathrm{a}} \pm 0.10$ \\
Mortalidad, \% & 6 & 3 & 3 \\
Índice de Eficiencia Productivo Europeo & 259 & 280 & 266 \\
\hline
\end{tabular}

${ }^{1}$ TO, T1, T2: 0,10 y $20 \%$ de harina de yuca en la dieta

${ }^{2}$ Promedios con superíndices iguales entre tratamientos no difieren estadísticamente Promedios \pm desviación estándar

La conversión alimenticia $(\mathrm{kg} / \mathrm{kg})$ fue estadísticamente similar en todos los tratamientos, tal y como ha sido reportado por Valdivié et al. (2008). Así mismo, el costo de la ración fue menor conforme se aumentó el nivel de harina de yuca, tomando en cuenta que el costo por kilogramo de harina de yuca fue de S/ 1.20 y el del maíz de S/ 1.50 (soles peruanos). Por otro lado, la mortalidad fue de 6,3 y $3 \%$ para T0, T1 y T2, respectivamente, principalmente por exceso de calor.

\section{Conclusión}

La inclusión de harina de yuca en reemplazo del maíz en la dieta de pollos parrilleros no afectó el comportamiento productivo de las aves.

\section{Literatura Citada}

1. Almeida J, Ferreira JR. 2005. Mandioca: uma boa alternativa para alimentação animal. Bahia agrícola 7: 50-56.

2. Brufau J, Pérez-Vendrell AM, Francesch M. 1994. Papel de la fibra en la alimentación avícola. En: Simposio de Avicultura. Pamplona, España.
3. Cagnon JR, Cereda MP, Pantalorto S. 2002. Glicósídeos cianogénicos da mandioca: biossíntese, distribuição, destoxificação e métodos de dosagem. Em: Cereda M (ed). Culturas de tuberosas amiláceas latino americanas. Sao Paulo, Brasil: Fundaçao Cargill. p 83- 99.

4. Cubas W, Córdova C, Jara W. 2009. Manejo agronómico del cultivo de maíz amarillo duro en selva baja. INIA. Folleto N. ${ }^{\circ}$ 3. 9 p. [Internet]. Disponible en: http://repositorio.inia.gob.pe/bitstream/inia/174/1/Manejo_agronomicomaiz_amarillo_duro_2009.pdf

5. Ferreira AHC. 2010. Raspa integral da raíz de mandioca para frangos de corte. Tese de mestrado. Piauí, Brasil: Universidade Federal do Piauí. 90 p.

6. Gonzales JJ. 2014. Efecto del lactosuero suministrado en forma líquida en dosis altas (30\%, 40\% y 50\%) en la crianza de pollos broilers en etapa de pollipavo (39 a 70 días). Tesis de Ingeniero Agrónomo. Tarapoto, Perú: Univ. Nacional de San Martín. 122 p.

7. Jones GP y Taylor RD. 2001. The incorporation of whole grain into pelleted broiler chicken diets: Production and physiological response. Br Poult Sci 42: 477-483. doi: 10.1080/00071660120070587 
8. Nascimiento GA, Costa FGP, Amarante Jr VS, Varros RL. 2005. Efeitos da substituição do milho pela raspa de mandioca na alimentação de frangos de corte, durante as fases de engorda e final. Cienc Agrotec 29: 200-207. doi: 10.1590/S141370542005-000100025

9. Rostagno H, Teixeira Albino L, Donzele J, Gomez, P, Oliveira R, Lopes D, Ferreira Al, et al. 2011. Brazilian tables for poultry and swine. $3^{\text {rd }}$ ed. Vicosa, Minas gerais, Brazil: Univ Federal de Vicosa. 251 p.
10. Silva JR, Ribeiro F, Bomfim, MD, Siqueira C. 2015. Avaliação nutricional do farelo de mandioca para frangos de crescimento lento em diferentes idades. Arch Zootec 64: 425-431. doi: 10.21071/ az.v64i248.430

11. Valdivié M, Leyva C, Cobo R, Ortiz A, Dieppa O, Febles M. 2008. Sustitución total del maíz por harina de yuca (Manihot esculenta) en las dietas para pollos de engorde. Rev Cubana Cienc Agríc 42: 61-64. 